Article

\title{
Hydrodynamic Efficiency Analysis of a Flexible Hydrofoil Oscillating in a Moderate Reynolds Number Fluid Flow
}

\author{
Paul Brousseau ${ }^{1, *(\mathbb{D})}$, Mustapha Benaouicha ${ }^{1,2}(\mathbb{D})$ and Sylvain Guillou ${ }^{1}(\mathbb{D}$ \\ 1 Cherbourg University Laboratory of Applied Sciences LUSAC, University of Caen Normandy, \\ 60 Rue Max-Pol Fouchet, 50130 Cherbourg-Octeville, France; mustapha.benaouicha@segula.fr (M.B.); \\ sylvain.guillou@unicaen.fr (S.G.) \\ 2 Segula Technologies, Research and Innovation Unit in Naval and Energy Engineering, \\ 9 Avenue Edouard Belin, 92500 Rueil-Malmaison, France \\ * Correspondence: paul.brousseau@unicaen.fr
}

Citation: Brousseau, P.; Benaouicha, M.; Guillou, S. Hydrodynamic Efficiency Analysis of a Flexible Hydrofoil Oscillating in a Moderate Reynolds Number Fluid Flow. Energies 2021, 14, 4370. https:// doi.org/10.3390/en14144370

Academic Editor: Eugen Rusu

Received: 9 June 2021

Accepted: 12 July 2021

Published: 20 July 2021

Publisher's Note: MDPI stays neutral with regard to jurisdictional claims in published maps and institutional affiliations.

Copyright: (c) 2021 by the authors. Licensee MDPI, Basel, Switzerland. This article is an open access article distributed under the terms and conditions of the Creative Commons Attribution (CC BY) license (https:// creativecommons.org/licenses/by/ $4.0 /)$.

\begin{abstract}
The paper focuses on the study of a semi-activated system, based on a combination of two movements of forced pitching and free-heaving motion. Therefore, quantifying with accuracy the hydrodynamic forces applied on the hydrofoil seems to be crucial. This is investigated throughout a numerical analysis of the hydrofoil dynamics. The deformable structure is oscillating in a lowReynolds number flow. In this study, a hydrofoil animated by a combined forced pitching and heaving movements is considered. Various materials of the hydrofoil structure are studied, from the rigid material to a more flexible one. A partitioned implicit coupling approach is applied in order to consider the Fluid-Structure Interaction (FSI) effects, while the Navier-Stokes equations are solved using the Arbitrary Lagrangian-Eulerian (ALE) method. Both the viscous incompressible Navier-Stokes equations and the elasticity equation are solved using finite volume method. The study is based on the analysis of the hydrodynamic loads acting on the structure. Therefore, the induced dynamics and the power coefficient of the structure are investigated. It is shown that the flexibility of the hydrofoil has an effect on its hydrodynamic behavior. Indeed it increases the load fluctuations and the horizontal mean force component. Furthermore, the unsteady vortices around the hydrofoil are highly impacted by its deformations. Finally, the structure deformations mostly improve the device energy efficiency.
\end{abstract}

Keywords: renewable marine energy; fluid-structure interactions; deformable hydrofoil; oscillating hydrofoil; NACA0015; CFD

\section{Introduction}

The wide variety of applications of oscillating structures in a fluid flow has drawn a lot of interest in the past decades. Numerous academic and industrial investigations have led to considerable advances in fluid dynamics and moving deformable structures [1]. Oscillating structures in a fluid flow can perform in two distinct modes: Power extraction and propulsion [2]. While the later has been widely investigated, among others, for aerodynamics or propulsion of flying and aquatic species [3], the former remains a recent application.

Recent interest in wind and tidal energies has led to various researches in order to understand the dynamics of tidal and wind turbines, and underlying physics [4-7]. Indeed, heaving and pitching movements of submerged structures have been the subject of numerical $[4,8,9]$ and experimental analysis $[3,5,10]$. These works were actively interested in optimizing the hydrodynamic performance of these structures, such as minimizing drag or maximizing their thrust or lift, depending on their use [2,11,12]. However, most of these studies are based on 2D modeling of immersed rigid solids. Therefore they do not 
take into account the Fluid-Structure Interactions (FSI) effects [1]. More recently, with the advances in computing resources and numerical coupling methods in FSI, realistic and efficient full-scale simulations are now possible [7].

Structure flexibility can enhance thrust and drag efficiency of a moving foil $[6,13]$. This behavior has been observed in natural phenomena and since then, studied for propulsion and energy extraction. Flexible flukes, for instance, boost the thrust efficiency of bottlenose dolphins by around 20\% [14]. Studies have shown that deformations of flexible structures must be precisely anticipated since they have a significant influence on structural performance [15]. Therefore, studies on the flexibility of blade materials have been carried out to improve the efficiency of wind and water turbines. For both technologies, flexible blades have been shown to act as a passive pitch control mechanism by making a dynamic adjustment and can prevent the stall effect [16]. Moreover, the flexibility of the blades provides higher thrust, lower normal forces, and minimal torque as well as reduced turbine vibrations, leading to higher efficiency and a significant increase of the self-starting capability $[6,15,16]$.

Monolithic and partitioned approaches are two of the most well-known numerical methods for handling FSI problems [17-19]. The monolithic method is more accurate than partitioned approaches and unconditionally stable [20]. It is, however, more difficult to implement. Therefore, it is often employed to solve simple FSI problems. Otherwise, partitioned methods are widely adopted in practice due to their ease of implementation. Indeed, by coupling with a dedicated interface, the conventional Computational Fluid Dynamics (CFD) and Computational Solid Dynamics (CSD) programs, they can be utilized for this purpose. In order to ensure the stability and accuracy of this approach for a wide range of applications, various coupling schemes have been developed. In addition, partitioned approaches allow the use of simplified models for the fluid or the structure, independently of one another. For instance, the slender structure can be modeled with beam theory $[21,22]$.

While FSI numerical approaches for analyzing the dynamic response of embedded deformable structures are commonly employed [23,24], taking into account, in addition to deformations, large free or forced displacements of the structure remains an unexplored field of investigation in the literature. Indeed, multiple sophisticated models are needed to acquire a reasonable description of the fluid and structural dynamics, as well as the dynamic mesh $[19,25]$. For any of these reasons, experimental investigations are more common in this situation. They are, however, still complex and expensive to set up $[3,5,11,26]$.

As previously shown, the efficiency of rotating wind and tidal turbines can be improved by using flexible blades. While numerous numerical and experimental investigations on rigid oscillating structures have been carried out in order to enhance their hydrodynamic performances, only few of them have investigated the fluid-induced deformations effects on these systems [27]. In addition, most of these works focused on the propulsive performances of the structure and not on the power extraction performances. For example, Alben [28] employed an analytical model to establish that the thrust power of a flexible and pitching structure has a series of resonant peaks as a function of its flexibility. David et al. [29] present an experimental work on the study of the thrust generation of a pitching and rigid foil with flexible flaps connected to its trailing edge. It has been established that the flexible flap significantly improves the thrust performance.

However, there is a lack of knowledge in the literature on the hydrodynamic performance of deformable heaving and pitching structures for energy extraction regime. For example, Yin and Luo [30] investigated the effects of the wing inertia for a range of wing stiffness on lift and drag performance and on energy consumption. It has been found that both inertia-induced deformations and flow-induced deformations can improve the lift of the structure. Furthermore, flow-induced deformations, as in the case of a low-mass wing, creates less drag and leads to greater aerodynamic power efficiency. Tian et al. [31] 
studied the effects of the flexibility of a flapping plate flow energy harvester. They present a strategy to enhance the energy extraction capability of the hydrofoil based on flapping plate active control.

Finally, while the fluid-induced deformations are considered in these investigation, the internal stresses and deformations of the structure are not resolved. Lately, Manjunathan and Bhardwaj [27] have conducted a numerical analysis in which the plate's internal stresses are resolved. This study showed that the propulsion is optimal for a specific pitching frequency. However, rigid plates outperform the flexible ones for large pitching frequency. The objective of this work is to carry out a numerical analysis of the FSI effects on a 2D flexible hydrofoil, subjected to a forced oscillating movement. A study of the impact of structure flexibility on hydrodynamic forces and on the efficiency of the hydrofoil are achieved.

In this paper, we first present the mathematical formulation of the problem in Section 2. Section 3 outlines the numerical methods applied to solve the problem. Then, the numerical validations and the obtained results are discussed in Section 4. Lastly, an overall conclusion is drawn in Section 5.

\section{Problem Formulation}

\subsection{Oscillating and Deformable Hydrofoil}

A moving and flexible hydrofoil immersed in a fluid flow is considered (Figure 1). The Reynolds numbers based on the hydrofoil's chord is $R e=2000$ (corresponding to the upstream velocity $U_{\infty}=0.002 \mathrm{~m} / \mathrm{s}$ ). The hydrofoil geometry is a 2D NACA0015 and its chord length is $c=1 \mathrm{~m}$. It oscillates sinusoidally, according to a forced pitching $\alpha(t)$ and heaving $h(t)$ motion, defined respectively by Equations (1) and (2):

$$
\begin{gathered}
\alpha(t)=\alpha_{0} \sin (\omega t) \\
h(t)=h_{0} \sin (\omega t+\varphi) .
\end{gathered}
$$

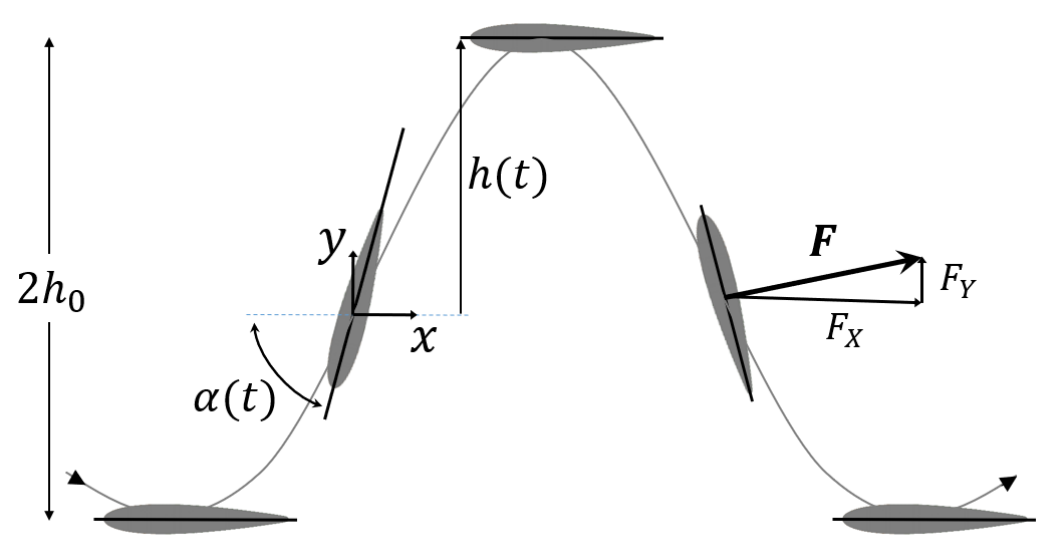

Figure 1. Oscillating hydrofoil in power extraction regime. Both the heaving $h(t)$ and the pitching $\alpha(t)$ motions are forced.

The center of rotation $O$ is located at $1 / 3$ of its chord (Figure 2). The heaving and pitching amplitudes are $\alpha_{0}$ et $h_{0}$ respectively. The angular frequency is $\omega=2 \pi f$ and $\varphi$ is the phase difference between the two motions. In the current study, $\varphi=90^{\circ}$ is kept constant. This choice is based on previous studies from the literature and ensures maximal efficiency $[2,32,33]$. Several pitching amplitudes are studied, from $\alpha_{0}=10^{\circ}$ to $30^{\circ}$ for one heaving amplitude corresponding to $h_{0} / c=0.43$. The oscillating frequency is $f^{*}=f h_{0} / U_{\infty}=0.025$. 


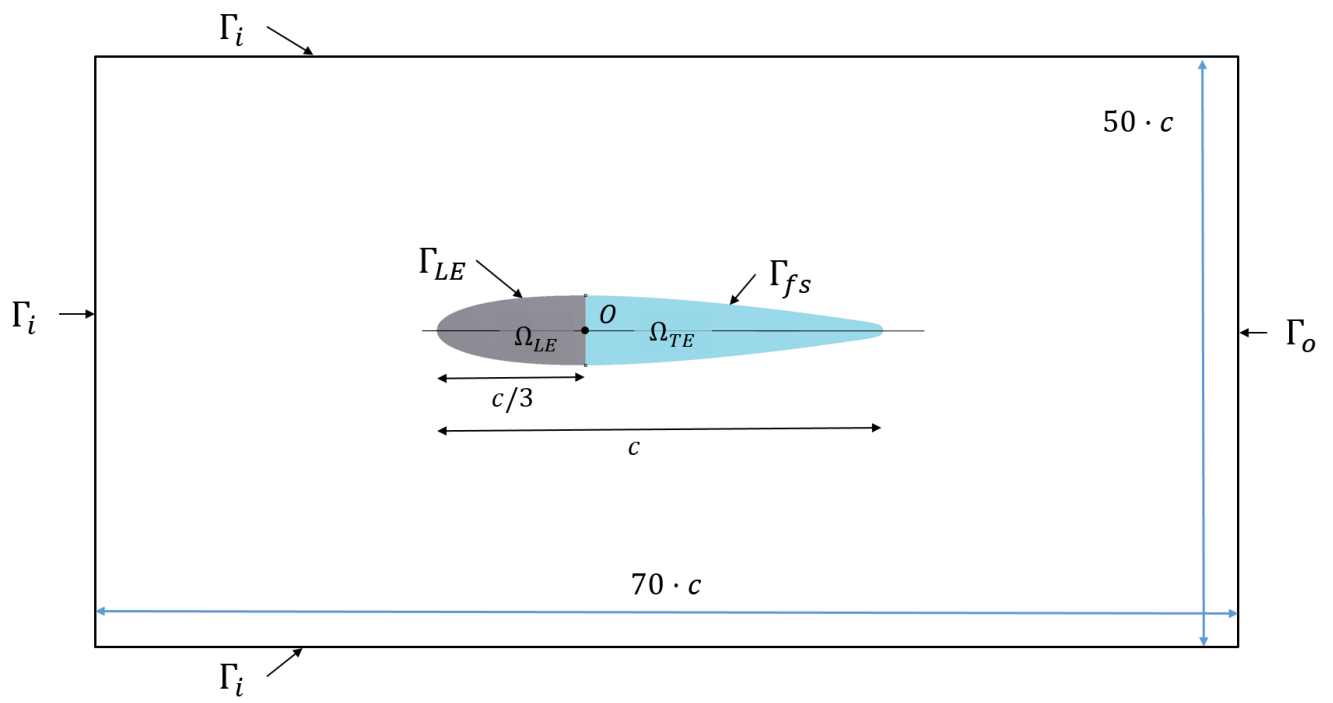

Figure 2. Partly deformable hydrofoil geometry, fluid, and solid domains boundaries: $\Omega_{L E}$ (gray) is non deformable and $\Omega_{T E}$ (cyan) is deformable.

The hydrofoil is made up of two parts. The first one, named $\Omega_{L E}$ (Figure 2), is considered as non-deformable, whereas the second one, $\Omega_{T E}$, deforms under the effect of the hydrodynamic forces. For this second part, three materials of different flexibilities, whose parameters are summarized in Table 1, are studied and compared.

Table 1. Mechanical characteristics of the hydrofoil material for the domain $\Omega_{T E}$.

\begin{tabular}{|c|c|c|c|}
\hline & Mat $_{1}$ & Mat $_{2}$ & $\mathrm{Mat}_{3}$ \\
\hline Designation $(-)$ & hydrofoil 1 & hydrofoil 2 & hydrofoil 3 \\
\hline Density $\rho_{s}\left(\mathrm{~kg} / \mathrm{m}^{3}\right)$ & 1420 & 1420 & 1420 \\
\hline Young's Modulus $E_{Y}(\mathrm{GPa})$ & 1.0 & 0.1 & 0.01 \\
\hline Poisson's Coefficient $v(-)$ & 0.35 & 0.35 & 0.35 \\
\hline
\end{tabular}

\subsection{Fluids Dynamics Equations}

The incompressible and unsteady flow dynamics is described by the Navier-Stokes equations (Equation (3)) given in Arbitrary Lagrangian-Eulerian (ALE) formulation.

$$
\left\{\begin{array}{rlrl}
\rho \frac{\partial \boldsymbol{u}}{\partial t}+\rho\left[\left(\boldsymbol{u}-\boldsymbol{u}_{g}\right) \cdot \nabla\right] \boldsymbol{u} & =-\nabla p+\mu \Delta \boldsymbol{u}-\rho \ddot{\boldsymbol{h}} \\
\nabla \cdot \boldsymbol{u} & =0 & \\
\boldsymbol{u} & =\boldsymbol{u}_{\infty} & & \text { on } \Gamma_{i} \\
\boldsymbol{u} & =\dot{\boldsymbol{\xi}} & & \text { on } \Gamma_{f s} \cap \Gamma_{L E} \\
\boldsymbol{u}_{t=0} & =\boldsymbol{u}_{\infty} & & \text { on } \Omega_{f} \\
p & =0 & & \text { on } \Gamma_{o}
\end{array}\right.
$$

with $u=(u, v)$ as the fluid velocity vector, $\boldsymbol{u}_{g}$ as the fluid domain velocity vector, $u_{\infty}=\left(U_{\infty}, 0\right), p$ as the fluid pressure, $\rho$ as the fluid density, and $\mu$ as the fluid viscosity. The unit normal vector $n$ points out of the fluid boundaries. $\dot{\xi}$ is the local structure velocity vector and the fluid domain boundaries $\Gamma_{i}, \Gamma_{0}$, and $\Gamma_{f_{S}}$ are respectively the inlet, the outlet, and the fluid-structure interface (Figure 2).

A heaving reference frame method is implemented. This choice is motivated by the desire to ease the mesh displacements. Therefore, no mesh displacements and deformations is required for modeling the large heaving displacement of the structure [2,34]. The 
reference frame is associated with the center of rotation of the structure. This leads to the addition of a driving force, reduced in this case to $\rho \ddot{h}$, where $\ddot{h}$ represents the vertical acceleration of the structure in the fixed reference frame. Thus, the heaving motion is taken into account by introducing a source term in the Navier-Stokes equations (Equation (3)) and imposing the condition $\boldsymbol{u}=\boldsymbol{u}_{\infty}-\dot{\boldsymbol{h}}$ on the domain boundaries, where $\boldsymbol{u}_{\infty}$ is the upstream velocity in the fixed reference frame.

Finally, the arbitrary velocity of the fluid domain is given by the solution of the diffusion equation (Equation (4)), where $d$ is the distance from the fluid-structure boundaries [35]:

$$
\left\{\begin{array}{rrll}
\nabla \cdot\left(\lambda \nabla \boldsymbol{u}_{g}\right) & =0 & \\
\boldsymbol{u}_{g} & =0 & \text { on } & \Gamma_{i} \cap \Gamma_{o} \\
\boldsymbol{u}_{g} & =\dot{\boldsymbol{\xi}} & \text { on } & \Gamma_{f \mathcal{S}} \cap \Gamma_{L E} \\
\lambda & =\frac{1}{d^{2}} & &
\end{array}\right.
$$

\subsection{Structure Dynamics Equations}

The following conservation equation (Equation (5)) describes the deformations of the structure.

$$
\left\{\begin{array}{rlr}
\rho_{s} \frac{\partial^{2} \xi}{\partial t^{2}} & =-\nabla \cdot \sigma_{s}+f_{s} & \\
\sigma_{f} \cdot n & =\sigma_{s} \cdot n & \\
\xi_{t=0} & =0 & \text { on } \Gamma_{f s} \cap \Gamma_{L E} \\
\dot{\xi}_{t=0} & =0 &
\end{array}\right.
$$

with $\rho_{s}$ as the structure mass density, $\xi$ as the structure local displacement vector, $\sigma_{S}$ as the Cauchy stress tensor, and $f_{s}$ as the body forces acting on the structure.

In the framework of the non-linear Saint Venant-Kirchhoff model, the conservation equation in Equation (5) can be written as follows [36]:

$$
\begin{aligned}
& \rho_{s} \frac{\partial^{2} \xi}{\partial t^{2}}= \nabla \cdot\left[\left(2 \mu_{s}+\lambda_{s}\right) \nabla \boldsymbol{\xi}\right]+ \\
& \nabla \cdot\left[-\left(\mu_{s}+\lambda_{s}\right) \nabla \xi+\mu_{s} \nabla^{T} \xi+\right. \\
&\left.\lambda_{s} \operatorname{tr}(\nabla \boldsymbol{\xi}) \boldsymbol{I}\right]+ \\
& \nabla \cdot\left[\mu_{s}\left(\nabla \boldsymbol{\xi} \cdot \nabla^{T} \boldsymbol{\xi}\right)+\right. \\
&\left.\frac{1}{2} \lambda_{s} \operatorname{tr}\left(\nabla \boldsymbol{\xi} \cdot \nabla^{T} \boldsymbol{\xi}\right) \boldsymbol{I}\right]+ \\
& \\
& \nabla \cdot[\boldsymbol{\Sigma} \cdot \nabla \boldsymbol{\xi}]
\end{aligned}
$$

where the stress tensor $\Sigma$, the strain tensor $E$, and the Lame's coefficients are given by the Equations (7)-(9) respectively [36]:

$$
\begin{gathered}
\boldsymbol{\Sigma}=2 \mu_{s} \boldsymbol{E}+\lambda_{s} \operatorname{tr}(\boldsymbol{E}) \boldsymbol{I} \\
\boldsymbol{E}=\frac{1}{2}\left(\nabla \boldsymbol{\xi}+\nabla^{T} \boldsymbol{\xi}+\nabla \boldsymbol{\xi} \cdot \nabla^{T} \boldsymbol{\xi}\right) \\
\mu_{s}=\frac{E_{Y}}{2(1+v)} \quad \lambda_{s}=\frac{v E_{Y}}{(1+v)(1-2 v)} .
\end{gathered}
$$

\subsection{Fluid-Structure Interaction Coupled Problem}

Equations (10) and (11) define the FSI coupling conditions. These conditions are required at the fluid-structure interface for the fluid and structure dynamic equations (Equations (3) and (5)) respectively [23].

$$
\begin{gathered}
\sigma_{f} \cdot n_{\mid \Gamma_{f s}}=\sigma_{s} \cdot n_{\mid \Gamma_{f s}} \\
\boldsymbol{u}_{\mid \Gamma_{f_{s}}}=\dot{\boldsymbol{\xi}}_{\mid \Gamma_{f s}}
\end{gathered}
$$


where, $\boldsymbol{u}_{\mid \Gamma_{f s}}$ and $\dot{\boldsymbol{\xi}}_{\mid \Gamma_{f s}}$ are respectively the fluid and solid velocity fields at the fluid-structure interface $\Gamma_{f s}$.

\section{Numerical Resolution}

A second order finite-volume discretization is used to solve the fluid equations (Equation (3)) with the Foam-Extend library while the time discretization for both fluid and structure equations is carried out with an implicit and second-order accurate scheme. The pressure-velocity coupling is resolved with the PISO algorithm, which requires a Courant number less than 1 .

The numerical code used to solve the structure dynamics equations is based on the finite volume discretization method [24,36-39].

The FSI coupled problem is solved using an implicit coupling scheme (Figure 3). The coupling convergence is defined by $\left\|\boldsymbol{r}_{i}\right\|_{2}<\varepsilon_{F S I, t o l}$. For each coupling iteration $i$, the residual vector $\boldsymbol{r}_{i}$ is defined at the fluid-structure interface (Equation (12)). Here, $\varepsilon_{F S I, t o l}=10^{-6}$ is the chosen convergence criteria.

$$
r_{i}=d_{\mid \Gamma_{f s}, i}-\xi_{f s, i}
$$

where, $\boldsymbol{d}_{\mid \Gamma_{f s}, i}$ and $\boldsymbol{\xi}_{f s, i}$ are the fluid and solid meshes displacements at the fluid-structure interface respectively. Further details about this method can be found in the literature $[13,36,40]$.

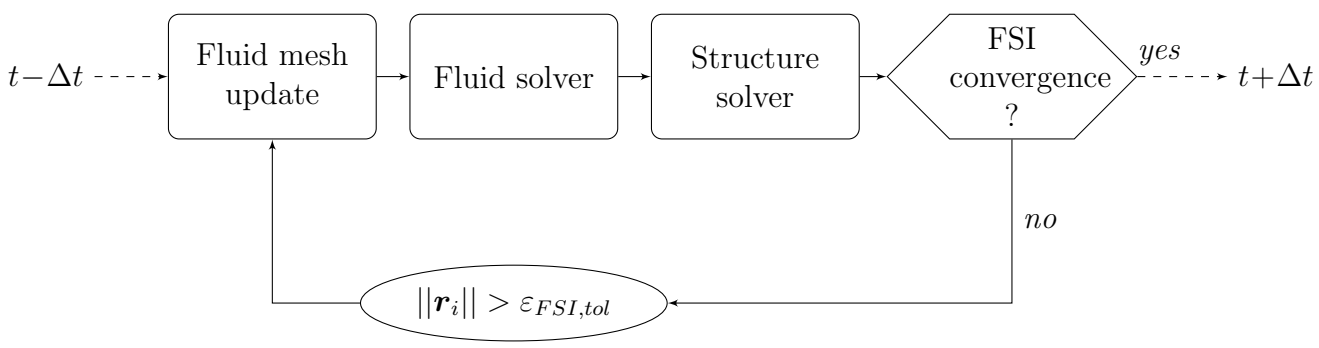

Figure 3. FSI implicit coupling scheme.

\subsection{Heaving Reference Frame Validation}

The study of a non-deformable 2D cylinder in a forced sinusoidal heaving motion is then performed in order to validate the structure heaving motion model. In this case, the flow regime is considered as laminar with a Reynolds number of $R e=500$, depending on the cylinder diameter $D=1 \mathrm{~m}$. The heaving amplitude is $\Delta h=2 \cdot h_{0}$ with $h_{0} / D=0.25$ and the dimensionless oscillation frequency is $f^{*}=f D / U_{\infty}=0.228$. A relative deviations of about $3.75 \%$ and $0.84 \%$ are obtained respectively for the mean drag coefficient $\left\langle C_{D}\right\rangle$ and the maximal lift coefficient $\widehat{C_{L}}$ compared to the results of Blackburn and Henderson [34]. Thus, a good agreement on the force predictions is achieved (Table 2). Therefore, this heaving modeling strategy is employed for the whole study.

Table 2. Comparison of mean drag coefficient $\left\langle C_{D}\right\rangle$ and peak lift coefficient $\widehat{C_{L}}$ for a non-deformable heaving cylinder obtained with the spectral element method [34] and CFD code predictions [2], for $R e=500, f^{*}=0.228$, and $h_{0} / D=0.25$.

\begin{tabular}{ccccc}
\hline Solvers & Cells & Time Steps/Cycle & $\left\langle\boldsymbol{C}_{\boldsymbol{D}}\right\rangle$ & $\widehat{\boldsymbol{C}_{\boldsymbol{L}}}$ \\
\hline Blackburn \& Henderson [34] & 422 & 2000 & 1.414 & 1.776 \\
Kinsey \& Dumas [2] & 65,600 & 2000 & 1.412 & 1.755 \\
Present & 75,000 & 2000 & 1.467 & 1.761 \\
Relative deviations with [34] & - & - & $3.75 \%$ & $0.84 \%$ \\
\hline
\end{tabular}




\subsection{FSI Implicit Coupling Scheme Validation}

First, the validation of the FSI implicit coupling scheme available in the OpenFoam FSI package is carried out $[13,36,38]$. Thus, the FSI problem of a 3D deformable cantilevered NACA0015 immersed in a turbulent fluid flow is solved and analyzed. This study is based on an experimental work carried out in a hydrodynamic channel [41]. The fluid-induced deformations of the structure are analyzed. The hydrofoil's angle of attack is $\alpha=8^{\circ}$, its chord is $c=100 \mathrm{~mm}$, and its spanwise dimension is $e=191 \mathrm{~mm}$. The flow is considered as turbulent with a Reynolds number of $R e=3.0 \cdot 10^{5}$. The lift and drag coefficients, $C_{L}$ and $C_{D}$ respectively, the angle of attack deviation $\Delta \alpha$ between the embedded and the free face of the hydrofoil, as well as the maximum leading edge displacement $d_{y}$ are summarized in Table 3. Numerical results obtained with the Openfoam code are compared to experimental data [41] and to numerical results obtained with the commercial code Ansys [23]. A relative deviation of less than $10 \%$, compared to experimental data, is obtained for the lift coefficient, the angle of attack variation, as well as the maximal deformation. On the other hand, the drag coefficient value is overestimated compared to the experimental one. The corresponding relative deviation is about $22 \%$. However, the same deviation is obtained for the same test case in the literature [23]. This large error could be explained by the use of the turbulence model $k-\varepsilon$. Overall, a good agreement with the experimental data is achieved.

Table 3. Numerical and experimental hydrodynamic coefficients $C_{L}$ et $C_{D}$, angle of attack deviation $\Delta \alpha$, and maximal strain $d_{y}$ for a 3D deformable cantilevered NACA0015. The relative deviations are based on the numerical results.

\begin{tabular}{ccccc}
\hline & $C_{\boldsymbol{L}}(-)$ & $C_{\boldsymbol{D}}(-)$ & $\Delta \boldsymbol{\alpha}\left({ }^{\circ}\right)$ & $d_{\boldsymbol{y}}[\mathrm{mm}]$ \\
\hline Experiment [41] & 0.80 & 0.045 & 0.2 & 1.48 \\
Numerical-Ansys [23] & 0.85 & 0.058 & 0.17 & 1.5 \\
Numerical-OpenFoam & 0.74 & 0.058 & 0.22 & 1.35 \\
Relative deviations with [41] & $7.5 \%$ & $22 \%$ & $10 \%$ & $8.5 \%$ \\
\hline
\end{tabular}

In order to develop a full heaving and pitching deformable hydrofoil model with the consideration of heaving and pitching motions, a preliminary model was implemented and validated. This focused on the modeling of a pitching deformable plate [40]. The same approach is employed in this study to model the pitching motion of the hydrofoil. Therefore, it is possible to analyze the fluid-induced deformations of the structure while forcing its motion. In the present work, the previous study is extended by adding the heaving motion to the pitching deformable hydrofoil.

\subsection{Mesh and Time Step Convergence Analysis}

Fluid's domain meshing influence on the numerical results is investigated. The energy efficiency $\eta$ is calculated for the pitching amplitudes $\alpha_{0}=10^{\circ}, 15^{\circ}$, and $15^{\circ}$ (that are outlined in Section 2.1), using four different mesh refinements. The hydrodynamic power $C_{P}$ (Equation (14)) and thus the energy efficiency $\eta$ (Equation (13)) are calculated from $C_{Y}$ (Equation (15)) and $C_{m}$ (Equation (16)):

$$
\begin{gathered}
\eta=\overline{C_{P}} \frac{c}{h_{0}} \\
C_{P}=C_{Y} \frac{\dot{h}}{U_{\infty}}+C_{m} \frac{\dot{\alpha} c}{U_{\infty}} \\
C_{Y}=\frac{F_{Y}}{\frac{1}{2} \rho S U_{\infty}^{2}} \\
C_{M}=\frac{M}{\frac{1}{2} \rho S c U_{\infty}^{2}} .
\end{gathered}
$$


It can be seen in Table 4 that from the space discretization Mesh 3 (Figure 4a), the numerical results remain the same and become independent of the fluid domain mesh. Indeed, the energy efficiency values do not evolve for a finer discretization. Therefore, this discretization is used for the rest of the study.

a)

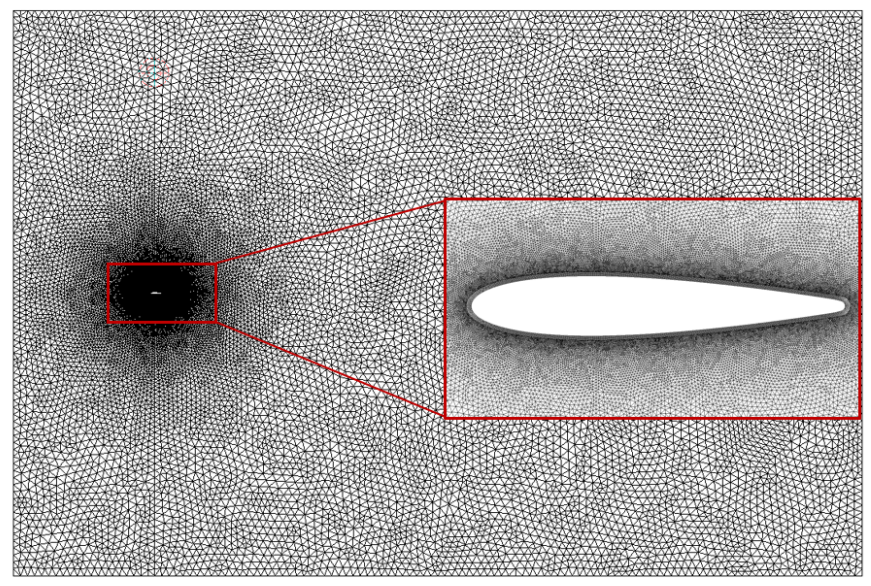

b)

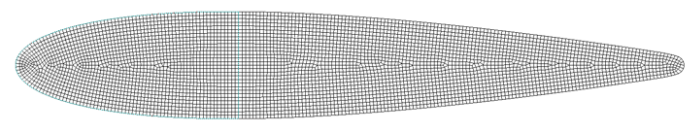

Figure 4. (a) Fluid mesh. (b) Structure mesh.

Table 4. Power efficiency $\eta(\%)$ for coarse to refine mesh for hydrofoil 1, $f^{*}=0.025$ and $\Delta h=0.8 \mathrm{~m}$, as a function of pitching amplitude $\alpha_{0}$.

\begin{tabular}{ccccc}
\hline & Mesh 1 & Mesh 2 & Mesh 3 & Mesh 4 \\
\hline$\alpha_{0}=10^{\circ}$ & 0.189 & 0.220 & 0.219 & 0.219 \\
$\alpha_{0}=15^{\circ}$ & 2.26 & 2.26 & 2.27 & 2.27 \\
$\alpha_{0}=20^{\circ}$ & 3.91 & 3.72 & 3.75 & 3.75 \\
\hline
\end{tabular}

The time step value used for the simulations corresponds to 2000 time steps per oscillating period. This choice is also employed in the literature [2,32].

Regarding the solid's domain discretization, the same mesh size used in [40] is employed in this paper (Figure $4 \mathrm{~b}$ ). This mesh density is considered as sufficient because smaller deformations are expected compared to those obtained in [40]. Indeed, the width-tolength ratio of the NACA0015 hydrofoil is twice as large as the one of the plate. Moreover, stiffer materials are used for the hydrofoil than for the plate.

\section{Results and Discussion}

\subsection{Analysis of the Flow in the Wake of the Hydrofoil}

Two characteristic behaviors of the flow can be observed with respect to the pitching amplitude of the hydrofoil. Indeed, for this specific oscillating frequency $\left(f^{*}=0.025\right)$, large pitching amplitude cases generate some dynamic-stall vortex shedding. Conversely, small pitching amplitude cases do not generate vortex. Both behaviors are illustrated by the instantaneous vorticity fields (Equation (17)) for the cases $\alpha_{0}=10^{\circ}$ and $\alpha_{0}=40^{\circ}$ respectively, represented in Figures 5 and 7 for the hydrofoils 1, 2, and 3. 
Hydrofoil 1

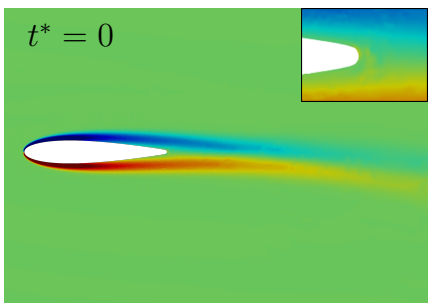

$t^{*}=0.25$

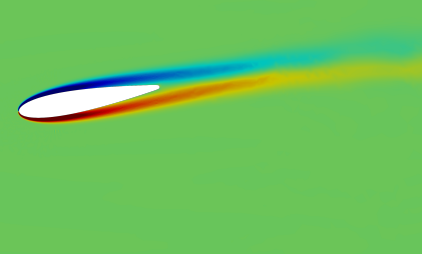

$t^{*}=0.5$

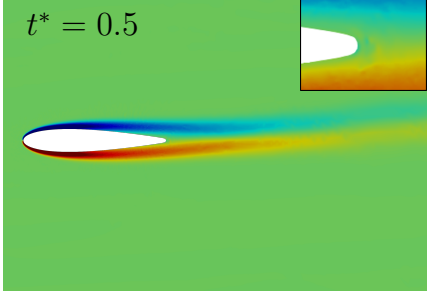

$t^{*}=0.75$

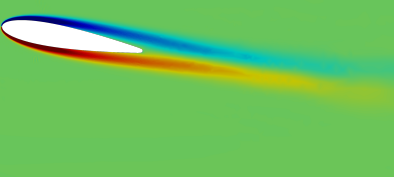

Hydrofoil 2
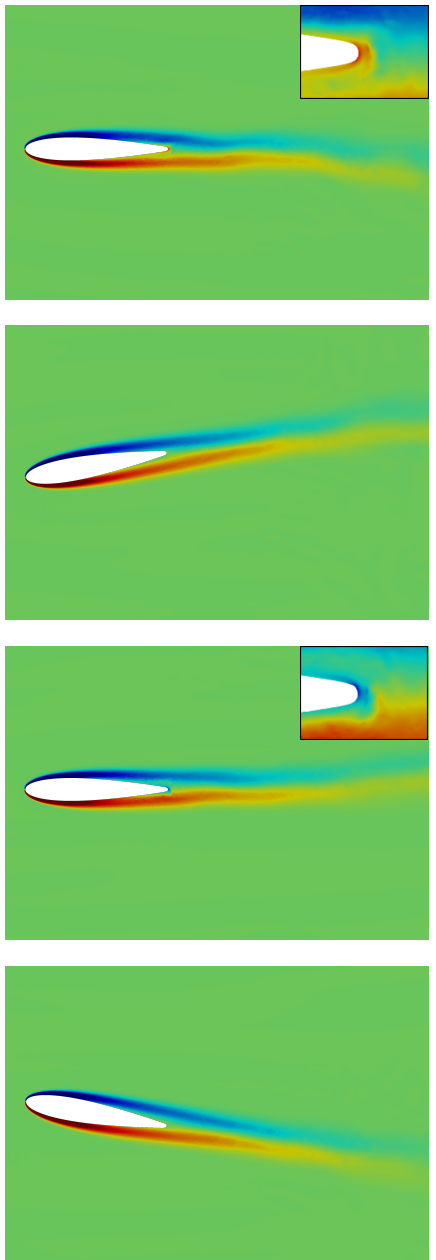

Hydrofoil 3
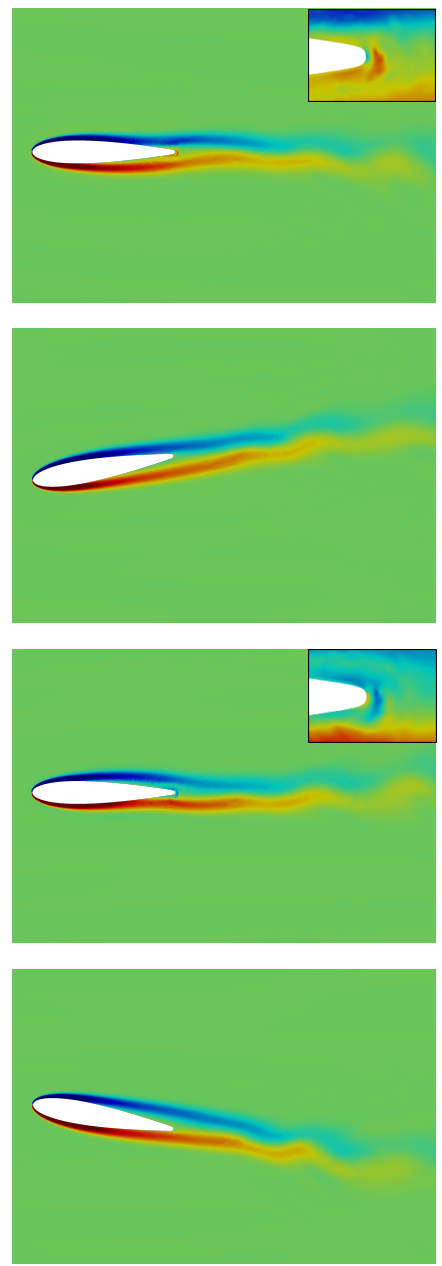

Figure 5. Instantaneous vorticity fields $\omega_{z}$ around the oscillating hydrofoil for the hydrofoils 1,2 , and $3, \alpha_{0}=10^{\circ}, f^{*}=0.025, \Delta h=0.8 \mathrm{~m}$, and $t^{*}=0,0.25,0.5,0.75$.

The snapshots are made at characteristic times $t^{*}=0,0.25,0.5,0.75$, defined as $t^{*}=(t-n T) / T$ where $n=0,1,2,3, \ldots$ and $T$ corresponds to the period number and the period value respectively:

$$
\omega_{z}=\frac{\partial v}{\partial x}-\frac{\partial u}{\partial y} .
$$

For a small pitching amplitude $\left(\alpha_{0} \leq 20^{\circ}\right)$, no vortex is generated in the flow by the hydrofoil dynamics, as seen in Figure 5. This applies to all flexible materials of the hydrofoil. For a non-deformable hydrofoil under the same conditions, the similar flow behavior has already been observed and discussed in the literature [2]. Indeed, it is shown that this is typical for a low oscillation frequency (in our case, $f^{*}=0.025$ ) and a low pitching amplitude.

For this case, the flow around the hydrofoil is slightly affected by the flexibility of the hydrofoil materials. However, small differences do appear and can be seen in the vorticity fields (Figure 5). These differences are imperceptible along the leading edge of the structure up to about $2 / 3$ of its chord. This is because the first third of the structure 
is non-deformable from the leading edge to its center of rotation, located at $1 / 3$ of the chord (Figure 2). On the other hand, notable differences appear from the last third of the hydrofoil, especially up to the trailing edge, and propagate into the wake. For $t^{*}=0$, a positive vorticity is generated at the trailing edge of the hydrofoils 2 and 3 cases. The generated vorticity is more important for the more flexible hydrofoil (hydrofoil 3), which is the most flexible hydrofoil. However, there is no vortex generation in the less flexible hydrofoil case (hydrofoil 1 ). The opposite phenomenon is observed for $t^{*}=0.5$, where a negative vorticity is generated at the trailing edge. This flow disturbance is then propagated into the wake and amplified according to the hydrofoil material flexibility. It is then more important in the case of hydrofoil 3.

Dynamic-stall vortex shedding appears when the pitching amplitudes increases. The vorticity amplitude increases with the hydrofoil pitching amplitudes, as shown in Figure 6 . For example, at $t^{*}=0.75$, a leading edge vortex is generated along the hydrofoil upper surface for $\alpha_{0} \geq 20^{\circ}$. In addition, for $\alpha_{0} \geq 25^{\circ}$, a trailing edge vortex is generated along the hydrofoil upper surface.

For the large pitching amplitude $\left(\alpha_{0} \geq 20^{\circ}\right)$, the flow along the hydrofoil surface, from the leading edge up to the $1 / 3$ of its chord, does not show any variation according to the three materials (Figure 7). This is also observed for the case of $\alpha_{0}=10^{\circ}$ and for all times. This is because of the rigidity of this part of the hydrofoil (see Figure 2).
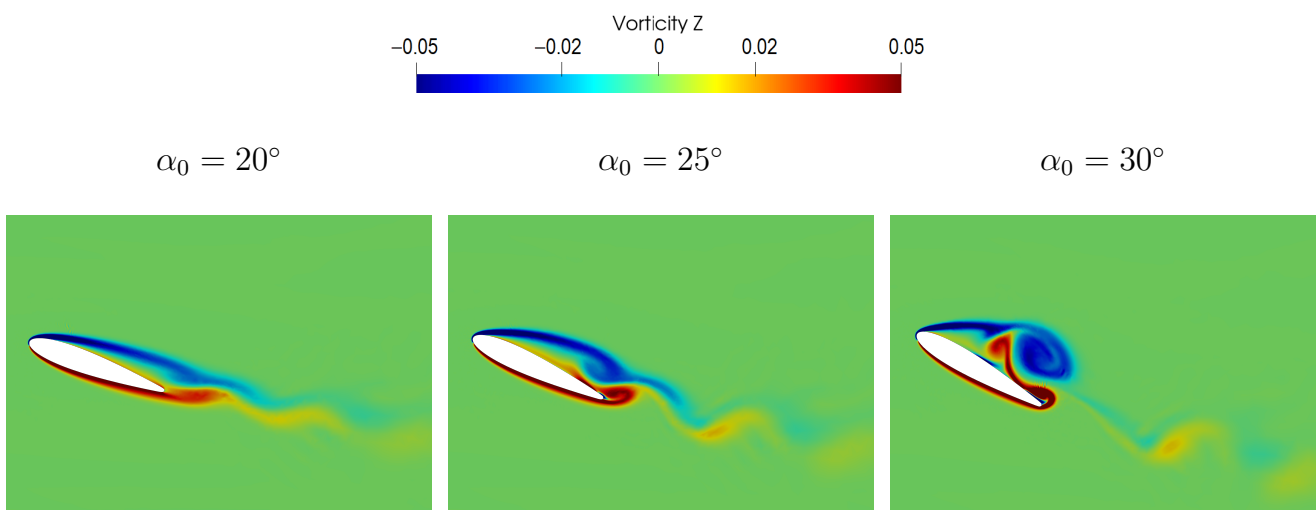

Figure 6. Instantaneous vorticity fields $\omega_{z}$ around the oscillating hydrofoil $2, \alpha_{0}=20,25$ and $30^{\circ}$, $f^{*}=0.025, \Delta h=0.8 \mathrm{~m}$, and $t^{*}=0.75$.

As shown previously, the material flexibility in the case of $\alpha_{0} \leq 20^{\circ}$ has a small impact on the flow dynamics. Conversely, for $\alpha_{0} \geq 20^{\circ}$, this has a significant impact. First, large vortices are now observed, due to the higher value of the angle of attack. At the time $t^{*}=0$, the flow along the upper surface of hydrofoil stalls earlier for the less flexible flexible hydrofoil (hydrofoil 1). Conversely, it never stalls for the most flexible hydrofoil (hydrofoil 3). On the lower surface, the opposite phenomenon occurs to a lesser extent. In fact, the flow almost stalls at the trailing edge for hydrofoil 3 while it never stalls for hydrofoil 2 and therefore for $=$ hydrofoil 1 . At $t^{*}=0.5$, a similar phenomenon, but symmetrical with respect to the chord, appears. In this case, the stall is observed along the lower surface of hydrofoils 1 and 2 and along the upper surface of hydrofoil 3.

Moreover, for $t^{*}=0.25$, a positive vortex is generated at the leading edge. The more flexible the hydrofoil material is, the faster the vortex is released. Indeed, at the same time, the vortex is completely detached from hydrofoil 3, while it is about to be released from hydrofoil 2 and is still attached to hydrofoil 1 . It is then advected by the flow. At $t^{*}=0.75$, the same phenomenon is observed on the extrados but a positive vortex becomes negative, and vice versa. 
Hydrofoil 1
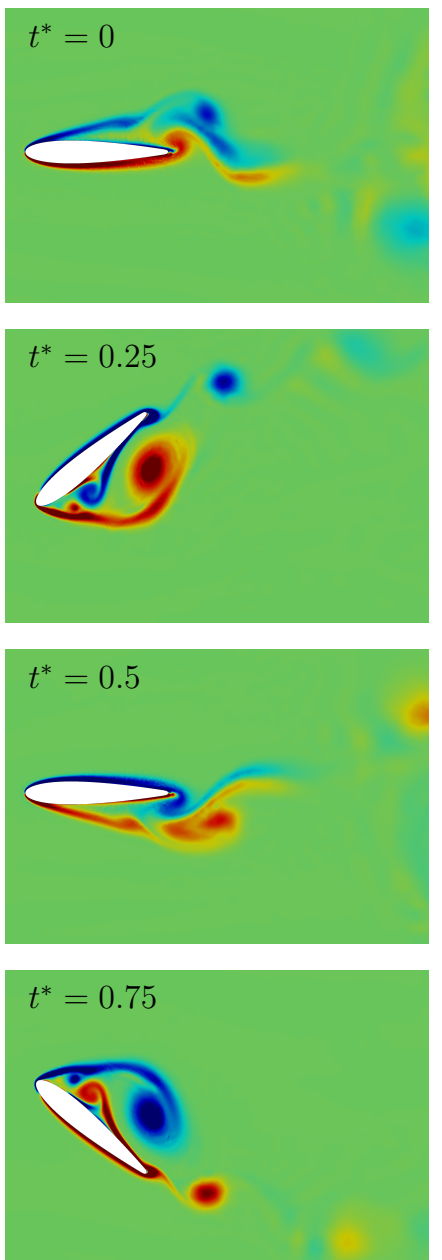

Hydrofoil 2
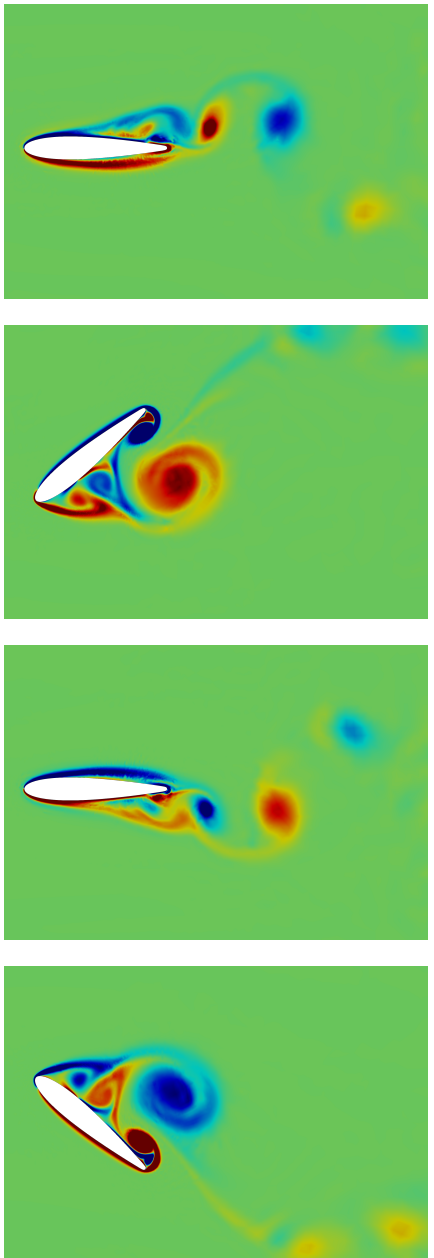

Hydrofoil 3
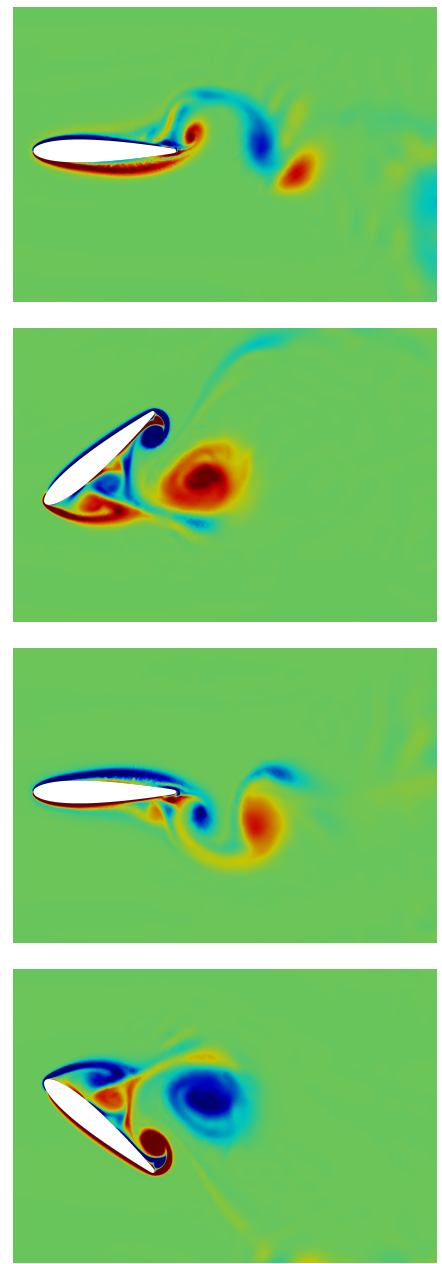

Figure 7. Instantaneous vorticity fields $\omega_{z}$ around the oscillating hydrofoil for the hydrofoils 1,2 , and $3, \alpha_{0}=40^{\circ}, f^{*}=0.025, \Delta h=0.8 \mathrm{~m}$, and $t^{*}=0,0.25,0.5,0.75$.

\subsection{Flexibility Influence on the Hydrodynamic Forces}

The qualitative fluid flow analysis in the previous section shows that the wake of the structure becomes increasingly disturbed as the flexibility of the hydrofoil increases. These flow disturbances has a significant impact on the hydrodynamic forces and therefore on the power extraction efficiency. The hydrodynamic coefficients $C_{X}$ (Equation (18)), $C_{Y}$ (Equation (15)), and $C_{M}$ (Equation (16)) are analyzed in this section. The pitching moment coefficient $C_{M}$ is calculated with respect to the structure center of rotation:

$$
C_{X}=\frac{F_{X}}{\frac{1}{2} \rho S U_{\infty}^{2}} .
$$

Figure 8 shows the time variations of hydrodynamic loads for the three hydrofoil materials and pitching amplitude ranging from $\alpha_{0}=10^{\circ}$ to $\alpha_{0}=40^{\circ}$. The hydrodynamic loads are almost periodic for all pitching amplitudes. Thus, a single oscillating period $T$ is represented. 

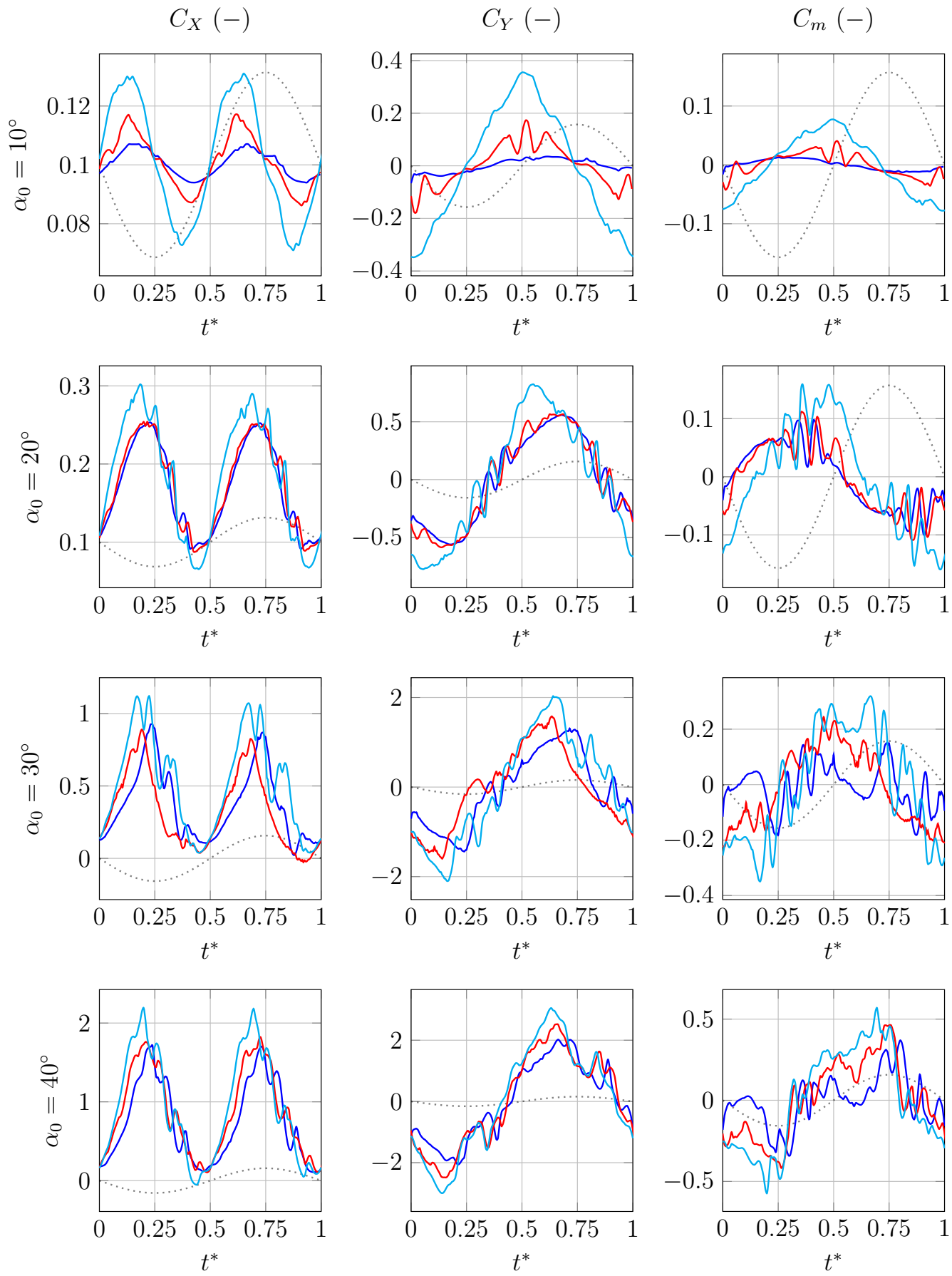

$\propto \dot{h} / U_{\infty} ;-$ Hydrofoil $1 ;-$ Hydrofoil $2 ;-$ Hydrofoil 3

Figure 8. Hydrodynamic coefficients $C_{Y}, C_{M}$, and $C_{X}$ and hydrofoil vertical velocity $\dot{h} / U_{\infty}$ versus time, for $\alpha_{0}=$ $10^{\circ}, 20^{\circ}, 30^{\circ}$, and $40^{\circ}$ and $f^{*}=0.025, \Delta h=0.8 \mathrm{~m}$ and for the three materials.

Firstly, for low pitching amplitude $\left(\alpha_{0}=10^{\circ}\right)$, the coefficient $C_{Y}$ remains relatively low (Figure 8). Nevertheless, the amplitude of $C_{Y}$ increases with the flexibility of the structure. A similar behavior is observed for the coefficient $C_{M}$. The average value of these two coefficients is almost zero for all materials. This can be explained by the fact that the heaving and the pitching motions are symmetrical. On the other hand, the mean value of the coefficient $C_{X}$ is equal to 0.1 for all the hydrofoils. However, the amplitude 
of this coefficient increases with the flexibility of the structure. This is probably due to the increase of the hydrofoil projected area in the plane perpendicular to the fluid flow direction. Finally, additional oscillations can be observed on the coefficient curve. This is due to the deformation of the structure.

For $\alpha_{0}=20^{\circ}$, the hydrodynamic coefficients of hydrofoils 1 and 2 are almost the same. However, the coefficient amplitudes for the most flexible hydrofoil (3) are larger than for hydrofoils 1 and 2. For this and a higher pitching amplitude, flexibility has less impact than for $\alpha_{0}=10^{\circ}$. However, for high pitching amplitudes, a phase advance of the coefficients is generated. For example, the maximum value of $C_{X}$ for $\alpha_{0} \geq 20^{\circ}$ is always obtained earlier for the most flexible materials.

There is a significant increase in the hydrodynamic forces for the pitching amplitude $\alpha_{0} \geq 30^{\circ}$ compared to $\alpha_{0} \leq 20^{\circ}$ (Figure 8). The mean value of the coefficient $C_{X}$ is also significantly higher for these cases. This is consistent with the vortex generation and the pitching amplitude increase.

Finally, for the pitching amplitude $\alpha_{0} \geq 30^{\circ}$, quite significant vibrations appear for all coefficients. This seems consistent with the appearance of vortices (Figure 7). These vibrations are maximal for $0.25<t^{*}<0.5$ and $0.75<t^{*}<1$, when the angle of attack is decreasing, from its maximum value $\left(10^{\circ}\right.$ to $\left.40^{\circ}\right)$ to its mean value $\left(0^{\circ}\right)$. It also occurs when the leading edge vortices break away from the structure. On the other hand, these disturbances tend to disappear for $0<t^{*}<0.25$ and $0.5<t^{*}<0.75$, when the angle of attack increases. Indeed, for $t^{*}=0$ and $t^{*}=0.5$, the vortices have been advected and are therefore already in the wake of the structure. These remarks are valid for all hydrofoils.

The horizontal force coefficient $C_{X}$ is not taken into account for the calculation of the energy extraction efficiency. However, mitigating the horizontal force component would reduce interactions with the hydrofoil support structures of the hydrofoil, and therefore enables more reliable and cheaper tidal turbines. The fluctuations of the horizontal forces have already been discussed (Figure 8). Here, the mean horizontal force coefficient $\overline{C_{X}}$ is plotted in Figure 9 for all pitching amplitudes $\alpha_{0}$. It can be seen that it increases with both the pitching amplitude $\alpha_{0}$ and the flexibility of the hydrofoil. Indeed, the more flexible the material, the larger the mean horizontal forces. This is true for all pitching amplitudes. However, for low pitching amplitudes $\left(\alpha \leq 25^{\circ}\right)$, the flexibility has very little effect on mean horizontal forces.

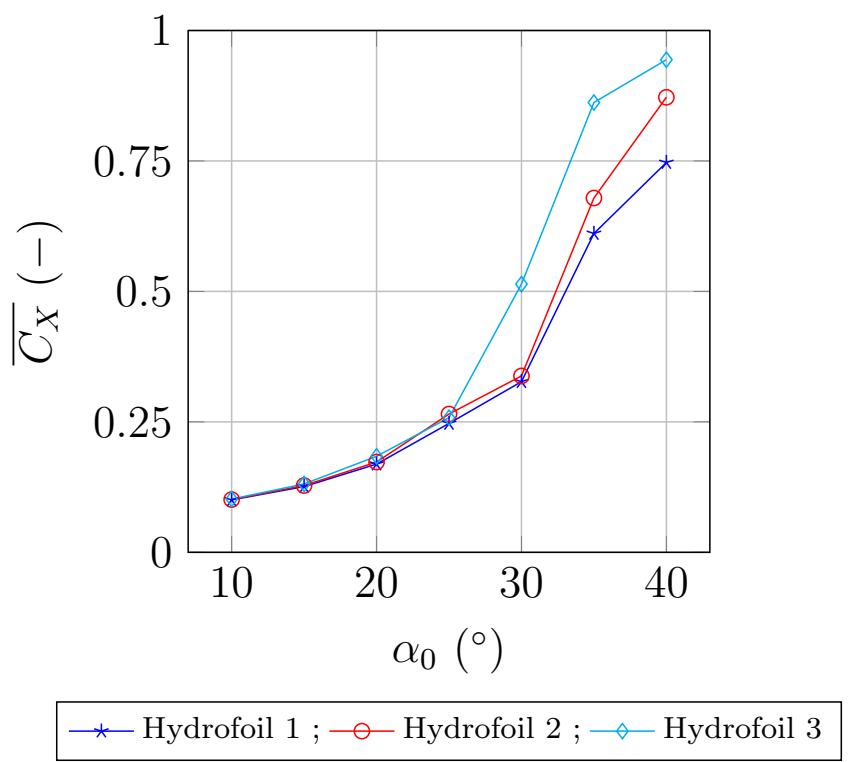

Figure 9. Mean hydrodynamic coefficient $\overline{C_{X}}$ versus pitching amplitude $\alpha_{0}$ for $f^{*}=0.025$, $\Delta h=0.8 \mathrm{~m}$, and for the three materials. 
Conversely, for a higher $\alpha_{0}$, the flexibilty effects are more visible and tend to increase the gap between the three hydrofoils. Finally, $\overline{C_{X}}$ increases linearly up to $\alpha_{0}=30^{\circ}$ for hydrofoils 1 and 2, while it increases linearly up to $\alpha_{0}=25^{\circ}$ for hydrofoil 3 .

\subsection{Flexibility Influence on the Power Extraction Efficiency of the Hydrofoil}

As shown in Figure 10, the instantaneous hydrodynamic power coefficient exhibits two distinct behaviors with respect to the pitching amplitudes. Firstly, for small pitching amplitude, such as $\alpha_{0}=10^{\circ}$ and $\alpha_{0}=20^{\circ}$, the power coefficient oscillates quasi-sinusoidally around its mean value. The higher the pitching amplitude, the higher the mean power coefficient value. Indeed, it is almost zero for $\alpha_{0}=10^{\circ}$ and around 0.04 for $\alpha_{0}=20^{\circ}$ (Figure 10). For a very low pitching amplitude, such as $\alpha_{0}=10^{\circ}$, the power coefficient amplitudes increase with the flexibility of the structure. Conversely, for a medium pitching amplitude $\left(\alpha_{0}=20^{\circ}\right)$, the power coefficient is less impacted by the flexibility. However, the flexibility tends to generate a phase lag, which is especially observable for hydrofoil 3 . This is also observed for a higher pitching amplitude such as $\alpha_{0} \geq 30^{\circ}$. For these large pitching amplitudes, the instantaneous power coefficient remains periodic but no longer oscillates sinusoidally. For hydrofoil 1 and 2, two peaks are observed at $t^{*}=0.25$ and $t^{*}=0.75$, corresponding to a maximum angle of attack.

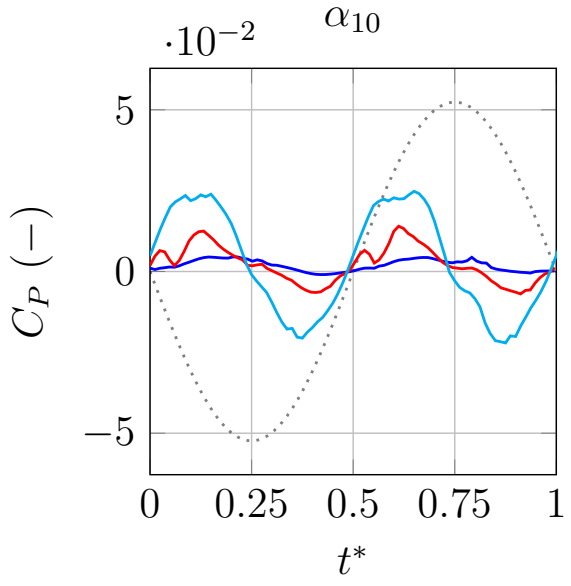

$\alpha_{30}$

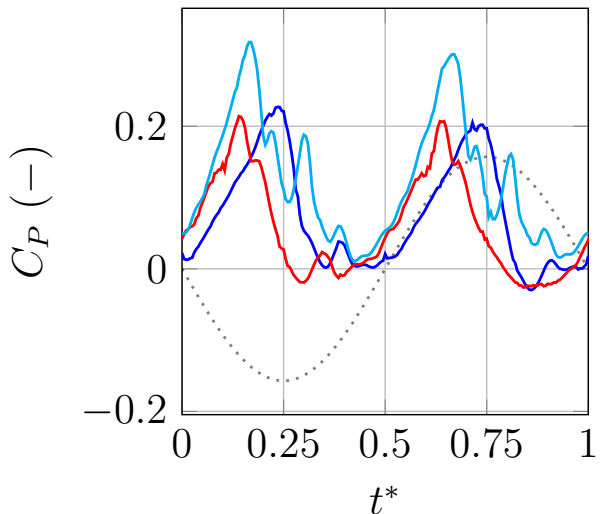

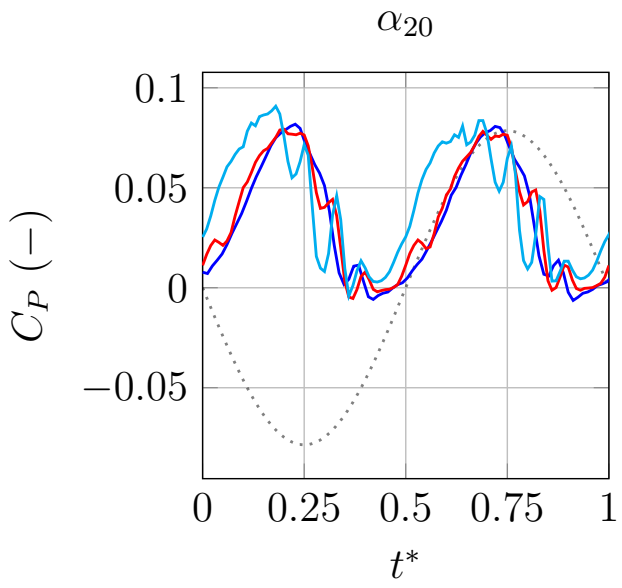

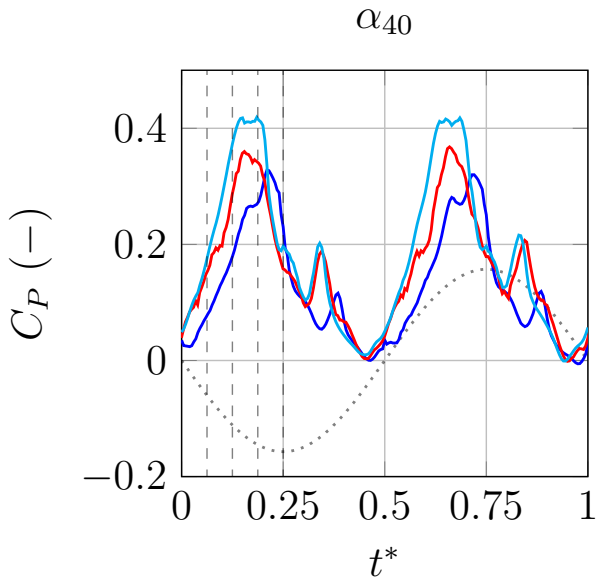

$\propto \dot{h} / U_{\infty} ;-$ Hydrofoil $1 ;-$ Hydrofoil $2 ;-$ Hydrofoil 3

Figure 10. Power coefficient $C_{P}$ and hydrofoil vertical velocity $\dot{h} / U_{\infty}$ versus time, for $\alpha_{10}$ to $\alpha_{40}$, $f^{*}=0.025, \Delta h=0.8 \mathrm{~m}$, and for three materials. 
Table 5 summarizes the power extraction efficiency for each hydrofoil and pitching amplitude. It can be seen that the efficiency increases with the pitching amplitude for all hydrofoils. Indeed, its value is very low ( $<1 \%$ ) for $\alpha_{0}=10^{\circ}$ and reaches a peak of $20.45 \%$ for hydrofoil 3 at $\alpha_{0}=40^{\circ}$.

Table 5. Power extraction efficiency $\eta(\%)$ for $f^{*}=0.025, \Delta h=0.8 \mathrm{~m}$, and for the three materials.

\begin{tabular}{cccc}
\hline & Hydrofoil 1 & Hydrofoil 2 & Hydrofoil 3 \\
\hline$\alpha_{0}=10^{\circ}$ & 0.2197 & 0.2567 & 0.3166 \\
$\alpha_{0}=15^{\circ}$ & 2.279 & 2.442 & 2.878 \\
$\alpha_{0}=20^{\circ}$ & 3.746 & 4.026 & 4.374 \\
$\alpha_{0}=25^{\circ}$ & 5.088 & 6.119 & 6.070 \\
$\alpha_{0}=30^{\circ}$ & 8.258 & 8.655 & 13.72 \\
$\alpha_{0}=35^{\circ}$ & 12.87 & 15.75 & 21.66 \\
$\alpha_{0}=40^{\circ}$ & 13.99 & 17.84 & 20.45 \\
\hline
\end{tabular}

Moreover, the hydrofoil flexibility has a significant impact on its power extraction efficiency. Overall, flexibility tends to increase the efficiency. However, two behaviors are noticeable. Indeed, the efficiency increases with the flexibility of the hydrofoil for the lowest and the highest pitching amplitudes. For example, it can be seen that for $\alpha_{0}=10^{\circ}$, the efficiency shows a relative improvement of $44 \%$ between hydrofoils 1 and 3 , while it shows a relative improvement of $46 \%$ for $\alpha_{0}=40^{\circ}$. For these pitching amplitudes, hydrofoil 2 generates a higher efficiency than hydrofoil 1 and a lower one than hydrofoil 3.

On the other hand, for moderate pitching amplitudes $20^{\circ} \leq \alpha_{0} \leq 30^{\circ}$, flexibility seems to have less impact on the efficiency. For example, there is no increase in power extraction efficiency between hydrofoils 2 and 3 for $\alpha_{0}=25^{\circ}$ and between hydrofoils 1 and 2 for $\alpha_{0}=30^{\circ}$.

\subsection{Fluid Pressure and Vorticity Fields Analysis}

As discussed previously, the power extraction coefficient $C_{P}$ and the horizontal force component are highly dependent on the hydrofoil material flexibility. It has been shown that the well-known leading edge vortex (LEV) phenomenon, which is frequently investigated when studying an oscillating structure in a fluid flow, can significantly increase the performances of oscillating foil [2]. In the present study, LEV phenomenon is observed for a pitching amplitude $\alpha_{0}$ greater or equal to $25^{\circ}$ and higher amplitudes. This is illustrated in Figures 6 and 11. Since the hydrofoil heaving displacements are symmetrical, it is expected to observe the same $C_{P}$ coefficient values during downward displacements $\left(0 \leq t^{*} \leq 0.5\right)$ as during upward ones $\left(0.5 \leq t^{*} \leq 1\right)$. Furthermore, it can be seen in Figure 10 that flexibility mainly influences the power coefficient in the first halves of the downward $\left(0 \leq t^{*} \leq 0.25\right)$ and upward $\left(0.5 \leq t^{*} \leq 75\right)$ heaving phase.

In Figure 11, the instantaneous vorticity and pressure fields are presented at four times, as marked in Figure 10. First, it is recalled that the vortex structures are similar in shapes for all the materials and for a specific pitching amplitude. This was already discussed in Section 4.1. Therefore, it is not surprising to observe similar pressure distributions around the hydrofoils. The pressure and vorticity around hydrofoil 1 (Figure 11a) give an example to understand the relationship between these flow fields and the power coefficient $C_{P}$. As the hydrofoil is pitching and heaving downward, a LEV is generated ( $t^{*}=0.1875$ ) along the downstream surface of the hydrofoil, along with a low pressure region. As the LEV is thickening, the power coefficient is increasing. Then, as it is convected by the fluid flow $\left(t^{*}=0.25\right)$, the power coefficient starts decreasing. 


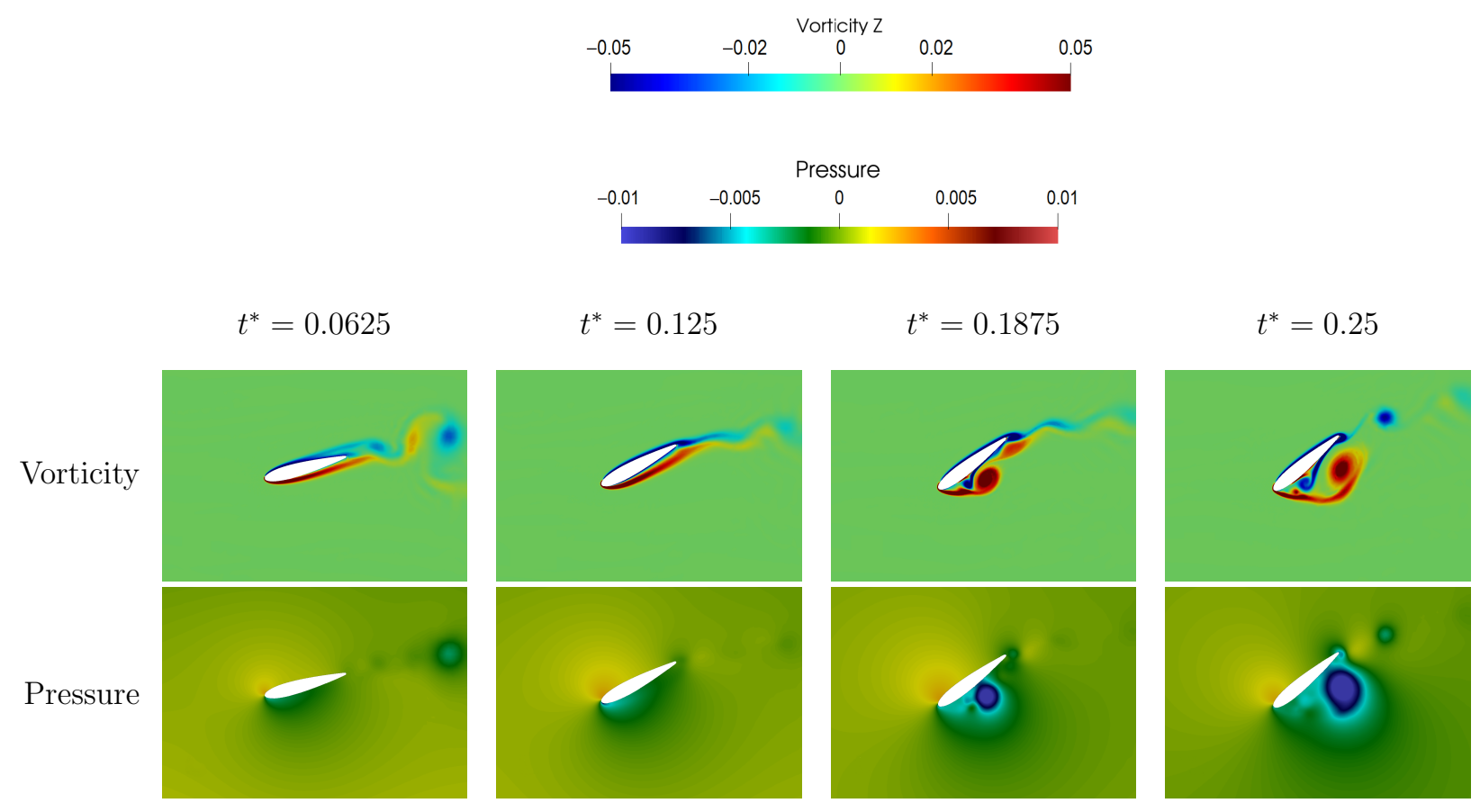

a) Hydrofoil 1
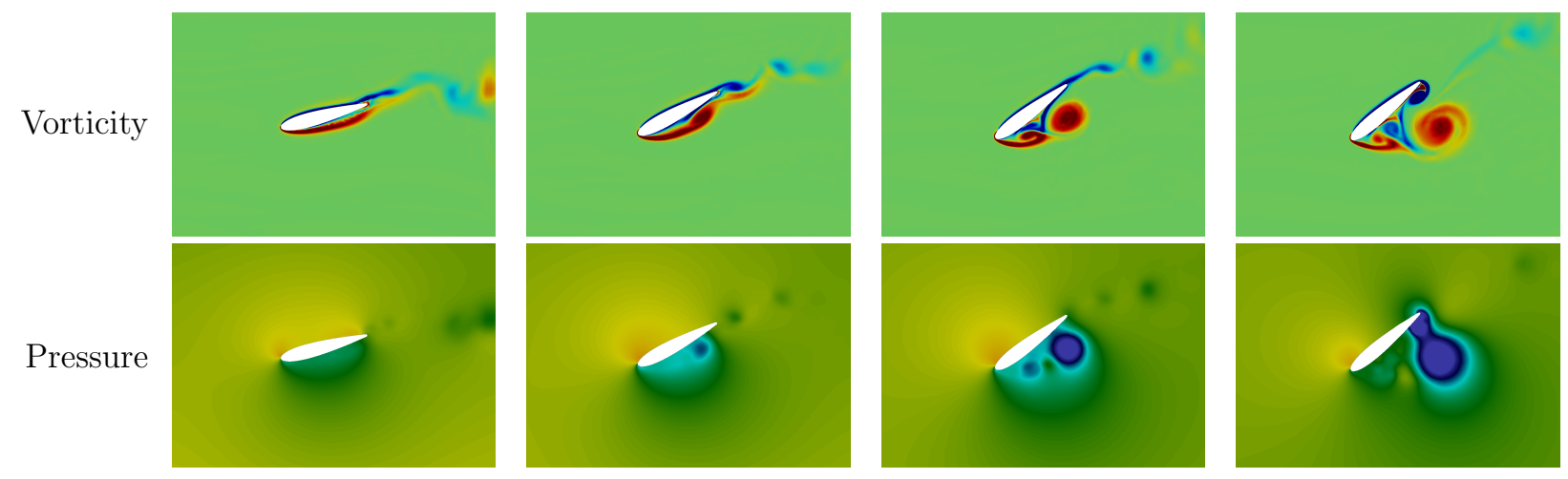

b) Hydrofoil 2
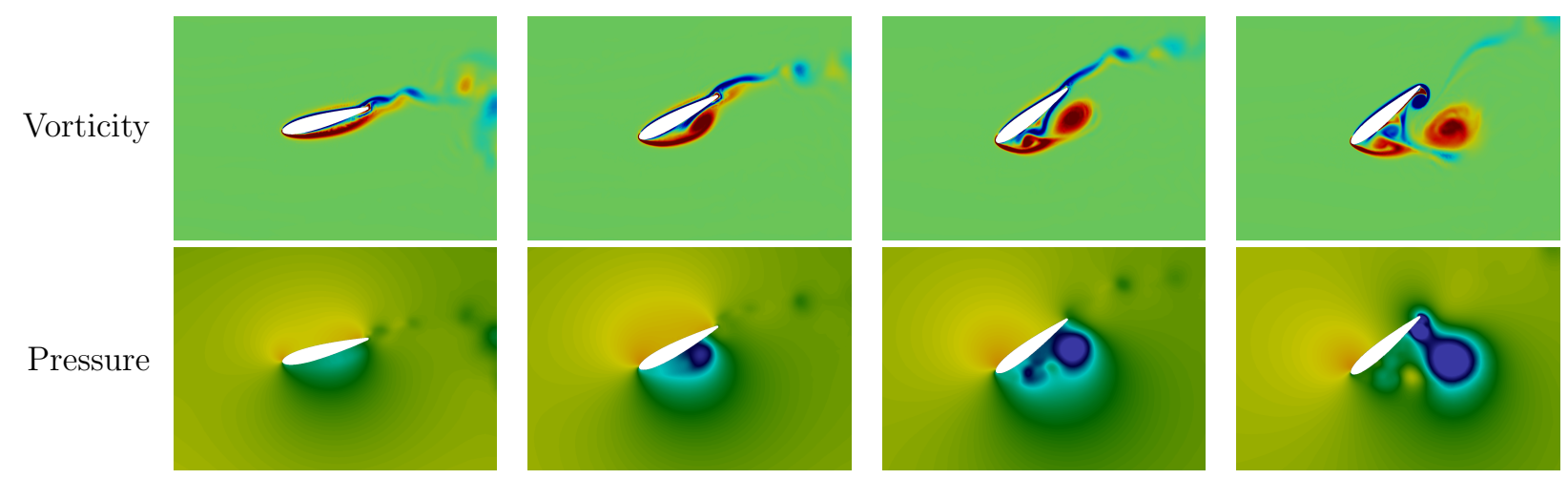

c) Hydrofoil 3

Figure 11. Vorticity and pressure fields around the oscillating hydrofoils (1-3) for $\alpha_{0}=40^{\circ}, f^{*}=0.025$, and $\Delta h=0.8 \mathrm{~m}$, at times $t^{*}=0.0625,0.125,0.1875$, and 0.25. 
The greater deformation of the more flexible hydrofoils (Figure 11b,c) causes a lower pressure load on the downstream surface and higher pressure load on the upstream surface of the hydrofoil. In addition, the LEV is generated and detached earlier. This phenomenon was also observed by Tian et al. [31]. This causes a higher maximum value of the power coefficient (Figure 10) for the more flexible structure. Furthermore, as the LEV appears earlier, the maximum value of $C_{P}$ is generated earlier. It is close to $t^{*}=0.1875$ and $t^{*}=0.125$ for hydrofoils 2 and 3 , respectively.

\section{Conclusions}

A numerical investigation of a deformable hydrofoil in forced heaving and pitching motion for energy production is presented in this study. The effects of both the material flexibility and the pitching amplitude on the hydrodynamic performances are investigated for one oscillation frequency and one heaving amplitude. The FSI effects are considered using a partitioned implicit coupling approach.

Thus, for this specific oscillation frequency, it has been shown that the efficiency is mostly improved with the flexibility of the structure. For a low pitching amplitude, no LEV has been observed. However, for higher pitching amplitudes, LEV occurs and has a significant impact on the hydrofoil performance. Indeed, the relationship between LEV, the low pressure region, and power extraction coefficient have been presented. Flexibility tends to lower the pressure drop and therefore to increase the power extraction coefficient. In addition, LEV stalls earlier for flexible material, which is coherent with the fact that the maximum $C_{P}$ value is earlier for the most flexible material. A maximum relative improvement of $46 \%$ between the most and least flexible hydrofoil has been obtained for $\alpha_{0}=40 \%$.

Finally, this study shows that the consideration of the hydrofoil deformations is necessary since it can have a huge benefit on its performances. This work should be completed by a complete parametric study taking into account different heaving amplitudes and different oscillation frequencies.

Author Contributions: Conceptualization, P.B., M.B. and S.G.; Data curation, P.B.; Formal analysis, P.B.; Funding acquisition, M.B. and S.G.; Investigation, P.B.; Methodology, P.B., M.B. and S.G.; Project administration, M.B. and S.G.; Resources, M.B. and S.G.; Software, P.B.; Supervision, M.B. and S.G.; Validation, P.B.; Visualization, P.B.; Writing—original draft, P.B.; Writing—review \& editing, P.B. All authors have read and agreed to the published version of the manuscript.

Funding: This research received no external funding.

Institutional Review Board Statement: Not applicable.

Informed Consent Statement: Not applicable.

Acknowledgments: This work was supported by ANRT CIFRE convention no. 2017/0927, with the collaboration of Segula Technologies. Most of this work was performed using the computing resources of CRIANN (Normandy, France). In addition, the authors would like to thank the Conseil Departemental de la Manche (CD50) for computational resources.

Conflicts of Interest: The authors declare no conflict of interest.

\section{Abbreviations}

The following abbreviations are used in this manuscript:

NACA National Advisory Committee for Aeronautics

LEV Leading-edge vortex

$\bar{a} \quad$ Mean value of $a$ over one motion period 


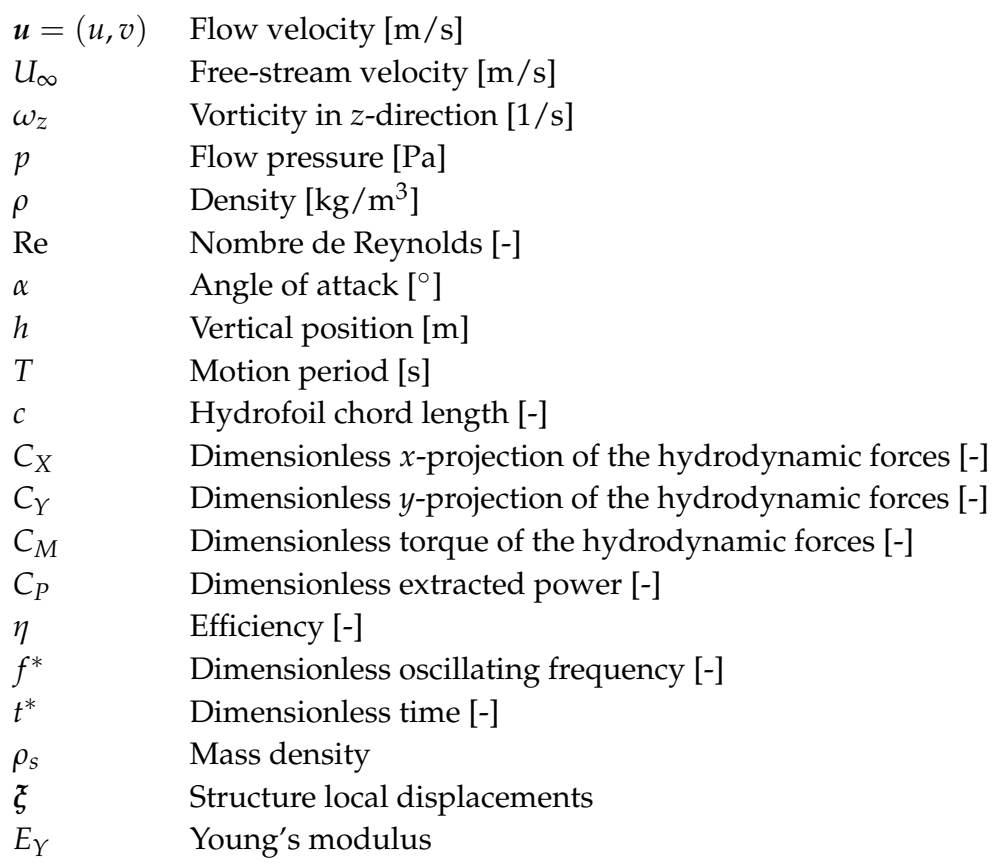

\section{References}

1. Xiao, Q.; Zhu, Q. A review on flow energy harvesters based on flapping foils. J. Fluids Struct. 2014, 46, 174-191. [CrossRef]

2. Kinsey, T.; Dumas, G. Parametric Study of an Oscillating Airfoil in a Power-Extraction Regime. AIAA J. 2008, 46, 1318-1330. [CrossRef]

3. Moubogha Moubogha, J.; Ehrenstein, U.; Astolfi, J.A. Forces on a pitching plate: An experimental and numerical study. Appl. Ocean. Res. 2017, 69, 27-37. [CrossRef]

4. Brousseau, P.; Benaouicha, M.; Guillou, S. Dynamics of a Free Heaving and Prescribed Pitching Hydrofoil in a Turbulent Flow, with a Fluid-Structure Interaction Approach. In Proceedings of the ASME 2018 Pressure Vessels and Piping Conference, Prague, Czech Republic, 15-19 July 2018; p. 9. [CrossRef]

5. Ducoin, A.; Astolfi, J.A.; Deniset, F.; Sigrist, J.F. An experimental and numerical study of the hydroelastic behavior of an hydrofoil in transient pitching motion. In Proceedings of the First International Symposium on Marine Propulsors, Trondheim, Norway, 22-24 June 2009.

6. Hoerner, S.; Abbaszadeh, S.; Maître, T.; Cleynen, O.; Thévenin, D. Characteristics of the fluid-structure interaction within Darrieus water turbines with highly flexible blades. J. Fluids Struct. 2019, 88, 13-30. [CrossRef]

7. Korobenko, A.; Yan, J.; Gohari, S.M.I.; Sarkar, S.; Bazilevs, Y. FSI Simulation of two back-to-back wind turbines in atmospheric boundary layer flow. Comput. Fluids 2017, 158, 167-175. [CrossRef]

8. Karbasian, H.R.; Esfahani, J.A. Enhancement of propulsive performance of flapping foil by fish-like motion pattern. Comput. Fluids 2017, 156, 305-316. [CrossRef]

9. Delafin, P.L.; Deniset, F.; Astolfi, J.; Hauville, F. Performance Improvement of a Darrieus Tidal Turbine with Active Variable Pitch. Energies 2021, 14, 667. [CrossRef]

10. Kinsey, T.; Dumas, G. Testing and Analysis of an Oscillating Hydrofoils Turbine Concept. In Proceedings of the ASME 2010 3rd Joint US-European Fluids Engineering Summer Meeting: Volume 1, Symposia-Parts A, B, and C, Montreal, QC, Canada, 1-5 August 2010; pp. 9-22. [CrossRef]

11. Duarte, L.; Dellinger, N.; Dellinger, G.; Ghenaim, A.; Terfous, A. Experimental investigation of the dynamic behaviour of a fully passive flapping foil hydrokinetic turbine. J. Fluids Struct. 2019, 88, 1-12. [CrossRef]

12. Marchand, J.B.; Astolfi, J.A.; Bot, P. Discontinuity of lift on a hydrofoil in reversed flow for tidal turbine Application. Eur. J. Mech. B 2017, 63, 90-99. [CrossRef]

13. Brousseau, P. Modélisation Numérique D'une Structure Oscillante en Tangage et Pilonnement, Avec Prise en Compte de L'interaction Fluide-Structure. Ph.D. Thesis, Université de Caen Normandie, Caen, France, 2020.

14. Fish, F.E. Power Output and Propulsive Efficiency of Swimming Bottlenose Dolphins (tursiops Truncatus). J. Exp. Biol. 1993, 185, 179-193. [CrossRef]

15. MacPhee, D.W.; Beyene, A. Fluid-structure interaction analysis of a morphing vertical axis wind turbine. J. Fluids Struct. 2016, 60, 143-159. [CrossRef]

16. Zeiner-Gundersen, D.H. A novel flexible foil vertical axis turbine for river, ocean, and tidal applications. Appl. Energy 2015, 151, 60-66. [CrossRef] 
17. Benaouicha, M. Contribution au Développement D'algorithmes de Couplage en Interaction Fluide-Structure. Ph.D. Thesis, Université de La Rochelle, La Rochelle, France, 2007.

18. Van Loon, R.; Anderson, P.D.; van de Vosse, F.N.; Sherwin, S.J. Comparison of various fluid-structure interaction methods for deformable bodies. Comput. Struct. 2007, 85, 833-843. [CrossRef]

19. Yan, J.; Augier, B.; Korobenko, A.; Czarnowski, J.; Ketterman, G.; Bazilevs, Y. FSI modeling of a propulsion system based on compliant hydrofoils in a tandem configuration. Comput. Fluids 2016, 141, 201-211. [CrossRef]

20. Michler, C.; Hulshoff, S.J.; van Brummelen, E.H.; de Borst, R. A monolithic approach to fluid-structure interaction. Comput. Fluids 2004, 33, 839-848. [CrossRef]

21. Arora, N.; Kang, C.K.; Shyy, W.; Gupta, A. Analysis of passive flexion in propelling a plunging plate using a torsion spring model. J. Fluid Mech. 2018, 857, 562-604. [CrossRef]

22. Olivier, M.; Dumas, G. Effects of mass and chordwise flexibility on 2D self-propelled flapping wings. J. Fluids Struct. 2016, 64, 46-66. [CrossRef]

23. Benaouicha, M.; Guillou, S.; Santa Cruz, A.; Trigui, H. Fluid-Structure Interaction Approach for Numerical Investigation of a Flexible Hydrofoil Deformations in Turbulent Fluid Flow. In Proceedings of the ASME 2018 Pressure Vessels and Piping Conference, Prague, Czech Republic, 15-19 July 2018; Volume 4, p. 9. [CrossRef]

24. Brousseau, P.; Benaouicha, M.; Guillou, S. Etude Numérique d'un Hydrofoil Déformable dans un Écoulement Turbulent avec Foam-extend et Prise en Compte de L'intéraction Fluide-Structure. Actes Des J. L'Hydrodynamique 2018. 2018. Available online: http:/ / website.ec-nantes.fr/actesjh/images/16JH/Articles/JH2018_papier_11_Brousseau_et_al.pdf (accessed on July 2021)

25. Huang, Z.; Xiong, Y.; Xu, Y. The simulation of deformation and vibration characteristics of a flexible hydrofoil based on static and transient FSI. Ocean. Eng. 2019, 182, 61-74. [CrossRef]

26. Hoerner, S.; Kösters, I.; Vignal, L.; Cleynen, O.; Abbaszadeh, S.; Maître, T.; Thévenin, D. Cross-Flow Tidal Turbines with Highly Flexible Blades-Experimental Flow Field Investigations at Strong Fluid-Structure Interactions. Energies 2021, 14, 797. [CrossRef]

27. Manjunathan, S.A.; Bhardwaj, R. Thrust generation by pitching and heaving of an elastic plate at low Reynolds number. Phys. Fluids 2020, 32, 073601. [CrossRef]

28. Alben, S. Optimal flexibility of a flapping appendage in an inviscid fluid. J. Fluid Mech. 2008, 614, 355-380. [CrossRef]

29. David, M.J.; Govardhan, R.N.; Arakeri, J.H. Thrust generation from pitching foils with flexible trailing edge flaps. J. Fluid Mech. 2017, 828, 70-103. [CrossRef]

30. Yin, B.; Luo, H. Effect of wing inertia on hovering performance of flexible flapping wings. Phys. Fluids 2010, 22, 111902. [CrossRef]

31. Tian, F.B.; Young, J.; Lai, J.C.S. Improving power-extraction efficiency of a flapping plate: From passive deformation to active control. J. Fluids Struct. 2014, 51, 384-392. [CrossRef]

32. Kinsey, T.; Dumas, G. Optimal Operating Parameters for an Oscillating Foil Turbine at Reynolds Number 500,000. AIAA J. 2014, 52, 1885-1895. [CrossRef]

33. Usoh, C.; Young, J.; Lai, J.; Ashraf, M. Numerical Analysis of a Non-Profiled Plate for Flapping Wing Turbines. In Proceedings of the 18th Australasian Fluid Mechanics Conference, Launceston, Australia, 3-7 December 2012.

34. Blackburn, H.M.; Henderson, R.D. A study of two-dimensional flow past an oscillating cylinder. J. Fluid Mech. 1999, 385, 255-286. [CrossRef]

35. Souli, M.; Zolesio, J.P. Arbitrary Lagrangian-Eulerian and free surface methods in fluid mechanics. Comput. Methods Appl. Mech. Eng. 2001, 191, 451-466. [CrossRef]

36. Cardiff, P.; Karač, A.; De Jaeger, P.; Jasak, H.; Nagy, J.; Ivanković, A.; Tuković, Ž. An open-source finite volume toolbox for solid mechanics and fluid-solid interaction simulations. arXiv 2018, arXiv:1808.10736.

37. Jasak, H.; Weller, H.G. Application of the finite volume method and unstructured meshes to linear elasticity. Int. J. Numer. Methods Eng. 2000, 48, 267-287. [CrossRef]

38. Tukovic, Z.; Karač, A.; Cardiff, P.; Jasak, H.; Ivanković, A. OpenFOAM finite volume solver for fluid-solid interaction. Trans. Famena 2018, 42, 1-31. [CrossRef]

39. Rege, K.; Hjertager, B.H. Application of foam-extend on turbulent fluid-structure interaction. IOP Conf. Ser. Mater. Sci. Eng. 2017, 276, 012031. [CrossRef]

40. Brousseau, P.; Benaouicha, M.; Guillou, S. Fluid-structure interaction effects on the deformable and pitching plate dynamics in a fluid flow. Appl. Ocean. Res. 2021, 113, 102720. [CrossRef]

41. Lelong, A.; Astolfi, J.A.; Guiffaud, P. An Experimental Analysis of Hydroelastic Response of Flexible Ligthweigth Hydrofoils in Non Cavitating and Cavitating Flow. J. Fluids Eng. 2016, 140, 021116. [CrossRef] 